effective in inducing tolerance. A large-scale, clinical trial of feeding bovine retinal proteins to patients with posterior uveitis at NIH is due to report in the near future. However, oral administration requires huge amounts of purified protein, which is unlikely to be economically viable on a large scale. Furthermore, using proteins derived from the neural tissue of cows in the UK is unlikely to be acceptable to patients for the foreseeable future. However, recombinant antigens can be made and the smaller but equally effective doses required for nasal tolerisation would seem to be relevant and certainly hold promise for the future. Intriguingly, in experimental models, it has been shown that tolerance may be induced by giving the antigen in tear drops at an equivalent dose to the nasal route, ${ }^{11}$ and it may well be that we will be able to offer our patients therapy in the form of eye drops before too long.

The Rayne Institute

St Thomas' Hospital

London

\section{References}

1. Dakin R. Remarks on a cutaneous affection produced by certain poisonous vegetables. Am J Med Sci 1829;4:98-100.

2. Mowat AM. The regulation of immune responses to dietary protein antigens. Immunol Today 1987;8:93-8.
3. Mestechy J, Hubby S, Moldoveanu Z, et al. Induction of tolerance in humans: effectiveness of oral and nasal immunisation routes. Ann N Y Acad Sci 1996;778: 194-201.

4. Stephen H, Thompson G, Staines NA. Could specific oral tolerance be a therapy for autoimmune disease? Immunol Today 1990;11:396-9.

5. Weiner HL, Freidman A, Miller A, et al. Oral tolerance: immunologic mechanisms and treatment of animal and human organ-specific autoimmune diseases by oral administration of autoantigens. Annu Rev Immunol 1994;12:809-37.

6. Chen Y, Inobe J, Marks R, et al. Peripheral deletion of antigen-reactive $\mathrm{T}$ cells in oral tolerance. Nature 1995;376:177-80.

7. Weiner HL, Mackin GA, Matsui M, et al. Double blind pilot trial of oral tolerisation with myelin antigens in multiple sclerosis. Science 1993;259:1321-4.

8. Trentham DE, Dynesius-Trentham RA, Orav EJ, et al. Effects of oral administration of type II collagen on rheumatoid arthritis. Science 1993;261:1727-30.

9. Higgins PJ, Weiner HL. Suppression of experimental allergic encephalomyelitis by oral administration of myelin basic protein and its fragments. J Immunol 1988;140:440-5.

10. Sun J-B, Holmgren J, Czerkinsky C. Cholera toxin B subunit: an efficient transmucosal carrier-delivery system for induction of peripheral immunological tolerance. Proc Natl Acad Sci 1994;91:10795-9.

11. Dua HS, Donoso LA, Labison PR. Conjunctival instillation of retinal antigens induces tolerance. Ocular Immunol Inflam 1994;2:29-36.

\title{
CLINICOPATHOLOGICAL CORRELATIONS PROVIDE NEW INSIGHTS INTO THE PATHOGENESIS OF AGE-RELATED MACULAR DEGENERATION
}

The neovascular form of age-related macular degeneration (AMD) poses an important clinical dilemma for ophthalmologists, as the only proven treatment modality - laser photocoagulation - has been largely ineffective in preventing visual loss in the majority of patients suffering from this disease. ${ }^{1,2}$ Histopathological studies in this condition seek clues to its aetiology in the hope that better understanding will allow the development of alternative and more effective treatment strategies. These studies have shown that the most characteristic feature of neovascular AMD is the accumulation of debris, notably drusen, basal laminar deposit (abnormal material located between the plasma membrane and basal lamina of the retinal pigment epithelium (RPE)) and basal linear deposit (material located between the basal lamina of the RPE and the inner collagenous zone of Bruch's membrane) ${ }^{3-5}$ It has been hypothesised that these deposits obstruct the normal transfer of oxygen and metabolites from the choriocapillaris to the outer retina, with the prevailing tissue hypoxia causing the release of angiogenic factors which provoke the growth of what are termed choroidal neovascular membranes $(\mathrm{CNV}) .^{6} \mathrm{~A}$ number of investigators have observed collections of macrophages and multinucleated giant cells in close proximity to the outer layer of Bruch's membrane at locations where the diffuse debris is present. ${ }^{6-8}$ It has been suggested that these inflammatory cells have been recruited to remove the debris and in the process of so doing, damage Bruch's membrane. The interaction of the inflammatory cells with RPE cells creates the conditions for the release of proinflammatory cytokines ${ }^{9}$ and potent angiogenic factors $^{10}$ which set the scene for continuing inflammation and neovascularisation. The damaged Bruch's membrane is then easily breached by the new vessels.

In this issue of Eye, Sarks et al., using material derived from two patients with preclinical neovas- 
cular macular degeneration, examine the early cellular events associated with the formation of choroidal neovascular membranes and the subsequent invasion of the sub-pigment epithelial space by the neovascular complex. ${ }^{19}$ Based on their histopathological observations they argue that the separation of the outer layers of Bruch's membrane from the choroid by membranous debris and soft drusen is a crucial event in the pathogenesis of neovascular AMD. This separation could potentially cause oxygen concentrations to fall in the outer retina, resulting in the release of diffusible angiogenic factors which paradoxically would reach the choroid in highest concentrations where the RPE is still attached to Bruch's membrane. In support of this hypothesis, Sarks et al. show that erosion of Bruch's membrane by choroidal neovascular complexes often commences beneath small hard drusen which anchor the RPE to Bruch's membrane. They also provide compelling evidence to show that separation of RPE from Bruch's membrane by diffuse debris is an important prerequisite for the next step which is the spread of the neovascular complex in the subpigment epithelial space.

Sarks et al. also attempt to elucidate some of the hitherto unexplained angiographic features that are commonly noted in clinically recognisable neovascular AMD. They observe that the growing vascular tips of the CNV, which extend approximately $100 \mu \mathrm{m}$ beyond the main membrane, lack pericyte cover and are capped with fibrin and a thick basementmembrane-like material. They suggest that these tips would leak fluid more readily and would correspond to the scalloped edges which are often seen on angiography. They also note that the RPE overlying the edge of the membrane is hyperpigmented and multilayered, which would account for the dark halo of blocked fluorescence so often noticed around the CNV on angiography. ${ }^{12}$

Understanding the causation and mechanism of disease is a vital part in the strategy for the development of new and potentially more effective treatments. Nowhere will this be more important than in the management of macular degeneration, which is responsible for visual handicap in what is estimated conservatively to be approximately 16 million individuals worldwide. Clinicopathological correlations using tissues derived from patients with different morphological types of neovascular AMD are essential for furthering our understanding of the aetiology and pathogenesis of this complex visual disorder.

Division of Vision Sciences

Usha Chakravarthy

Queen's University and Royal Victoria Hospital

Belfast

Northern Ireland

\section{References}

1. Moissiev J, Alhahel A, Masuri R, Treister G. The impact of macular photocoagulation study results on the treatment of age related macular degeneration. Arch Ophthalmol 1995;113:185-9.

2. Yannuzzi LA. A new standard of care for laser photocoagulation of subfoveal choroidal neovascularization of age-related macular degeneration. Arch Ophthalmol 1994;112:462-6.

3. Green WR, Enger C. Age-related macular degeneration: histopathological studies. The 1992 Lorenz E. Zimmerman Lecture. Ophthalmology 1993;100: 1519-35.

4. Sarks JP, Sarks SH, Killingsworth MC. The evolution of soft drusen in age-related macular degeneration. Eye 1994;8:269-83.

5. Kuntz CA, Jacobson SG, Cideciyan AV, Li Z-Y, Stone EM, Possin D, Milam AH. Sub-retinal pigment epithelial deposits in a dominant late-onset retinal degeneration. Invest Ophthalmol 1996;37:1772-82.

6. Dastgheib K, Green WR. Granulomatous reaction to Bruch's membrane in age-related macular degeneration. Arch Ophthalmol 1994;112:813-8.

7. Killingsworth MC, Sarks JP, Sarks SH. Macrophages related to Bruch's membrane in age-related macular degeneration. Eye 1990;4:23-31.

8. Heriot WJ, Henkind P, Belhorn RW, Burns MS. Choroidal neovascularization can digest Bruch's membrane: a prior break is not essential. Ophthalmology 1984;91:1603-8.

9. Elner SG, Elner VM, Bian SM, Lukacs NW, Kurtz RM, Strieter RM, Kunkel SL. Human retinal pigment epithelial cell interleukin 8 and monocyte chemotactic protein 1 modulation by T-lymphocyte products. Invest Ophthalmol 1997;38:446-55.

10. Lopez PF, Sippy BD, Lambert M, Thach AB. Transdifferentiated retinal pigment epithelial cells are immunoreactive for vascular endothelial growth factor in surgically excised age-related macular degenerationrelated choroidal neovascular membranes. Invest Ophthalmol 1996;37:855-68.

11. Sarks JP, Sarks SH, Killingsworth MC. Morphology of early choroidal neovascularisation in age-related macular degeneration: correlation with activity. Eye 1997;11:515-22.

12. Lopez PF, Lambert HM, Grossniklaus HE, Sternberg P. Well-defined sub-foveal choroidal neovascular membranes in age-related macular degeneration. Ophthalmology 1993;100:415-22. 